\title{
DISPONIBILIDADE DE COBRE PARA MUDAS DE EUCALIPTO EM SOLOS DE CERRADO ${ }^{(1)}$
}

\author{
Fernando Antonio Vieira Rodrigues ${ }^{(2)}$, Nairam Félix de $\operatorname{Barros}^{(3)}$, \\ Júlio César Lima Neves ${ }^{(3)}$, Víctor Hugo Alvarez V. ${ }^{(3)}$ \& Roberto \\ Ferreira Novais ${ }^{(3)}$
}

\begin{abstract}
RESUMO
No Brasil, com a intensificação da silvicultura clonal, o plantio de materiais genéticos mais produtivos e exigentes nutricionalmente tem aumentado o aparecimento de sintomas de deficiência de micronutrientes, principalmente de $\mathrm{B}$ e, mais recentemente, de $\mathrm{Cu}$ e de $\mathrm{Zn}$, sobretudo em áreas de Cerrado. Este trabalho teve por objetivo avaliar três métodos de determinação da disponibilidade de Cu (Mehlich-1, Mehlich-3 e DTPA pH 7,3) para mudas de eucalipto cultivadas em casa de vegetação, em amostras de seis solos de Cerrado, e a influência de propriedades do solo na eficiência desses extratores. Os tratamentos foram arranjados no esquema fatorial 6 x 5 , correspondendo a seis solos e cinco doses de $\mathrm{Cu}\left(0,2,4,8\right.$ e $\left.16 \mathrm{mg} \mathrm{dm}^{-3}\right)$, com quatro repetições, em blocos ao acaso. Os níveis críticos de $\mathrm{Cu}$ em solos de Cerrado, para o crescimento de mudas de eucalipto, são iguais ou inferiores a 0,12 e $0,09 \mathrm{mg} \mathrm{dm}^{-3}$, pelos extratores Mehlich-1 e DTPA, respectivamente. Os teores e conteúdos de $\mathrm{Cu}$ na planta mostram estreita relação com os teores de $\mathrm{Cu}$ no solo pelos três extratores. Os teores de $\mathrm{Cu}$ pelo Mehlich-1, Mehlich-3 e DTPA são altamente correlacionados entre si. A avaliação da disponibilidade de $\mathrm{Cu}$ para mudas de eucalipto pode ser feita com qualquer um dos três extratores. Entretanto, o Mehlich-1 é o extrator mais recomendado, pela facilidade operacional deste método em relação aos outros e por ele já ser usado na grande maioria dos laboratórios do País.
\end{abstract}

Termos de indexação: disponibilidade, extratores, cobre, fertilização, eucalipto.

\footnotetext{
(1) Parte da Dissertação de Mestrado em Solos e Nutrição de Plantas do primeiro autor, na Universidade Federal de Viçosa - UFV. Recebido para publicação em setembro de 2009 e aprovado em setembro de 2010.

(2) Doutorando do Programa de Pós-Graduação em Solos e Nutrição de Plantas, Universidade Federal de Viçosa - UFV. Avenida P.H. Rolfs s/n, CEP 36571-000 Viçosa (MG). E-mail: fvrgaucho@hotmail.com

(3) Professor do Departamento de Solos, UFV. E-mails: nfbarros@ufv.br; julio_m2003@yahoo.com.br; vhav@ufv.br; rfnovais@ufv.br
} 


\title{
SUMMARY: COPPER AVAILABITY FOR EUCALYPTUS SEEDLINGS IN CERRADO SOILS
}

\begin{abstract}
In Brazil, along with the intensification of clonal forestry, the management of more productive and more nutrient-demanding genotypes has led to the appearance of micronutrient deficiency symptoms, mainly of Boron, and more recently, of Copper (Cu) and Zinc, especially in Cerrado areas. The purpose of this study was to evaluate three extractant solutions to determine Cu availability (Mehlich-1, Mehlich-3 and DTPA pH 7.3) for eucalyptus seedlings grown under greenhouse conditions in six Cerrado soil samples and to assess the influence of soil properties on the efficiency of these extractors. Treatments were arranged in a randomized block design, with four replications, in a $6 \times 5$ factorial scheme, representing six soils and five $\mathrm{Cu}$ doses $\left(0,2,4,8\right.$, and $\left.16 \mathrm{mg} \mathrm{dm}^{-3}\right)$. The critical Cu levels in Cerrado soils for eucalyptus seedlings, by the extractors Mehlich-1 and DTPA, are equal to or below 0.12 and $0.09 \mathrm{mg} \mathrm{dm}^{-3}$, respectively. All three extractors showed a close relationship of the $\mathrm{Cu}$ plant concentration and content to $\mathrm{Cu}$ soil concentration. The Cu concentrations were strongly correlated among Mehlich-1, Mehlich-3 and DTPA extractors. Consequently, Cu availability to eucalyptus seedlings can be evaluated using any of the three extractors. However, Mehlich-1 is the most recommended method. Firstly, due to the easy handling compared to other methods and, secondly, due to the widespread use in most routine analysis laboratories of Brazil.
\end{abstract}

Index terms: extractors, copper, fertilization, eucalyptus.

\section{INTRODUÇÃO}

No Brasil, com a intensificação da silvicultura clonal e o plantio de materiais genéticos mais produtivos e exigentes nutricionalmente, tem aumentado o aparecimento de sintomas de deficiência de micronutrientes, principalmente de B e, mais recentemente, de $\mathrm{Cu}$ e de $\mathrm{Zn}$, sobretudo em áreas de Cerrado. Até então, os programas de adubação de eucalipto voltavam-se ao suprimento de macronutrientes, com poucas empresas utilizando micronutrientes, à exceção do B (Pinheiro, 1999; Bouchardet, 2002).

A deficiência de $\mathrm{Cu}$ tem causado mais danos físicos às plantações, pois as árvores com esse problema apresentam galhos alongados e frágeis, que se quebram com facilidade, causando deformação da copa (Marschner, 1995; Dell et al., 2001). Observações de campo indicam que a ocorrência e a intensidade dos sintomas variam com o tipo de solo, a época do ano, o material genético, entre outros fatores, e que, de modo geral, esses sintomas desaparecem com o avanço da idade das plantas.

A avaliação da disponibilidade de nutrientes é feita, em geral, pelo uso de extratores químicos, sendo ideal aquele que extrai as formas absorvíveis pelas plantas e que apresenta adequação aos laboratórios de rotina. Além disso, os teores obtidos devem se correlacionar estreitamente com as respostas das culturas sem e com aplicação dos nutrientes no solo e em várias condições de ambiente e manejo (Bray, 1948). Dada a dificuldade de atender a todas essas premissas, estudos como este se fazem necessários.
Este trabalho teve por objetivo avaliar três métodos de determinação da disponibilidade de $\mathrm{Cu}$ (Mehlich-1, Mehlich-3 e DTPA pH 7,3) para mudas de eucalipto cultivadas em casa de vegetação, em amostras de seis solos do Cerrado, e a influência de propriedades do solo na eficiência desses extratores.

\section{MATERIAL E MÉTODOS}

Com base nos dados obtidos por Sequeira (2007), foram coletadas amostras de solos de áreas de plantios comerciais de eucalipto na região do Cerrado de Minas Gerais (Quadro 1).

A unidade experimental foi constituída por um vaso com $1,8 \mathrm{dm}^{3}$ de solo e duas mudas clonais de eucalipto (E. urophylla $\times$ E. grandis). Os tratamentos foram arranjados no esquema fatorial $6 \times 5$, correspondendo a seis solos e cinco doses de $\mathrm{Cu}$, com quatro repetições, em blocos ao acaso. As doses de $\mathrm{Cu}$ foram de 0, 2, 4, 8 e $16 \mathrm{mg} \mathrm{dm}^{-3}$, na forma de $\mathrm{CuCl}_{2} \cdot 2 \mathrm{H}_{2} \mathrm{O}$.

Além de $\mathrm{Cu}, 5$ dias antes do transplante, foi aplicada uma adubação básica, em $\mathrm{mg} \mathrm{dm}^{-3}$, constituída de: 100 de N, 300 de P, 165 de K, 70 de S, 0,15 de Mo, 2,0 de B, 5,0 de Zn e 4,0 de Mn (modificado de Alvarez V., 1974). Em cobertura, aos 20 e 35 dias após o transplante, foram aplicados $25 \mathrm{mg} \mathrm{dm}^{-3} \mathrm{de} \mathrm{N}$ e 0,88 $\mathrm{mg} \mathrm{dm}^{-3}$ de Fe em cada aplicação. Para os solos com teores de $\mathrm{Ca}^{2+}$ e $\mathrm{Mg}^{2+}$ menores do que 1,0 e $0,5 \mathrm{cmol}_{\mathrm{c}} \mathrm{dm}^{-3}$, respectivamente, foram aplicados cloretos de $\mathrm{Ca}$ e de $\mathrm{Mg}$ para atingir esses teores, de 
Quadro 1. Caracterização química e física dos seis solos utilizados no estudo

\begin{tabular}{|c|c|c|c|c|c|c|c|c|c|c|}
\hline Solo* & $\mathrm{pH} \mathrm{H}_{2} \mathrm{O}^{(1)}$ & $\mathrm{Ca}^{2+(2)}$ & $\mathrm{Mg}^{2+(2)}$ & $\mathrm{Al}^{3+(2)}$ & $\mathrm{H}+\mathrm{Al}^{(3)}$ & $\mathbf{P}^{(4)}$ & $\mathbf{K}^{(4)}$ & $\mathrm{MO}^{(5)}$ & P-rem ${ }^{(6)}$ & $\operatorname{Argila}^{(7)}$ \\
\hline & & 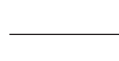 & $-\mathrm{cmol}$ & $\mathrm{m}^{-3}$ & 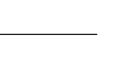 & $-\mathrm{mg} \mathrm{c}$ & $\mathrm{I}^{-3}-$ & dag kg-1 & $\mathrm{mg} \mathrm{L}^{-1}$ & $\%$ \\
\hline 1 & 4,82 & 0,20 & 0,08 & 0,75 & 5,00 & 64,8 & 14 & 2,05 & 37,3 & 20,1 \\
\hline 2 & 5,71 & 2,26 & 2,12 & 0,00 & 6,25 & 2,3 & 238 & 4,80 & 16,7 & 55,3 \\
\hline 3 & 5,49 & 0,91 & 1,05 & 0,24 & 5,98 & 0,6 & 92 & 3,30 & 13,9 & 60,7 \\
\hline 4 & 4,66 & 0,20 & 0,08 & 0,94 & 7,95 & 0,9 & 17 & 3,87 & 22,3 & 42,6 \\
\hline 5 & 4,92 & 0,31 & 0,31 & 0,68 & 5,60 & 0,5 & 46 & 2,91 & 24,2 & 56,1 \\
\hline 6 & 5,06 & 0,63 & 0,14 & 0,80 & 4,05 & 1,0 & 51 & 2,02 & 35,3 & 35,0 \\
\hline
\end{tabular}

(1) $\mathrm{pH}$ em água - relação 1:2,5. ${ }^{(2)} \mathrm{Ca}^{2+}, \mathrm{Mg}^{2+} \mathrm{e} \mathrm{Al}^{3+}$ : extrator $\mathrm{KCl} 1 \mathrm{~mol} \mathrm{~L}^{-1} \cdot{ }^{(3)} \mathrm{H}+\mathrm{AL}$ : extrator $\mathrm{Ca}(\mathrm{OAc})_{2} 0,5 \mathrm{~mol} \mathrm{~L}^{-1} \mathrm{pH} 7,0 .{ }^{(4)} \mathrm{P}$ e K : extrator Mehlich-1. ${ }^{\left({ }_{5}\right)}$ MO: C. org. x 1,724 -Walkley Black. ${ }^{(6)}$ P-rem: fósforo remanescente - Alvarez, V. et al. $\left(2000 .{ }^{\left({ }^{(7)}\right.}\right.$ Argila Ruiz (2005). * Origem dos solos: João Pinheiro (1), Vazante (2 e 3), Três Marias (4) e Curvelo (5 e 6).

modo que não se aumentasse o $\mathrm{pH}$ do solo. Para facilitar a homogeneização com todo o volume de solo, os adubos foram dissolvidos em água.

Os tratamentos foram aplicados num volume de solo de $2,1 \mathrm{dm}^{3}$, sendo colocados $1,8 \mathrm{dm}^{3}$ no vaso para o transplante das mudas, e uma alíquota de $300 \mathrm{~cm}^{3}$ foi incubada por 15 dias. No momento do transplante, as mudas, provenientes do enraizamento de miniestacas, tinham altura média em torno de $15 \mathrm{~cm}$. As raízes das mudas foram lavadas cuidadosamente com água, para remover porções do substrato eventualmente aderidas ao sistema radicular. Foi feita uma seleção das mudas por altura, separando-as em cada bloco. Os tratamentos foram aplicados em cada unidade experimental em separado. Durante todo o experimento, foi feito, semanalmente, o rodízio dos vasos.

Após o período de incubação, preparou-se a TFSA para análise dos teores de Cu pelos extratores Mehlich-1 (Mehlich, 1953), Mehlich-3 (Mehlich, 1984) e DTPA (Lindsay \& Norvell, 1978). A relação solo:extrator para o Mehlich-1 e Mehlich-3 foi de 1:10, com agitação a $200 \mathrm{rpm}$ por $5 \mathrm{~min}$ em agitador horizontal; os extratos foram pipetados após $16 \mathrm{~h}$ de repouso. Para o DTPA, a relação foi de 1:2, com agitação por $2 \mathrm{~h}$ no mesmo agitador e rotação, sendo o extrato filtrado logo em seguida. Após 70 dias, as plantas foram cortadas e secas em estufa a $65^{\circ} \mathrm{C}$ até atingirem peso constante. Posteriormente, foram pesadas, moídas e submetidas à digestão nítrico-perclórica. Nos extratos obtidos (solo e planta), foram determinadas as concentrações de $\mathrm{Cu}$ por espectrofotometria de absorção atômica.

Os teores de $\mathrm{Cu}$ obtidos pelos três métodos, bem como os respectivos teores e conteúdos na matéria seca da parte aérea (MSPA) das plantas, foram submetidos à análise de variância. $\mathrm{O}$ efeito da aplicação de doses de Cu sobre a produção de MSPA, os teores recuperados pelos três extratores e os teores e conteúdos na MSPA foi avaliado por meio de análise de regressão. Os teores disponíveis no solo pelos três métodos foram correlacionados entre si. As taxas de recuperação pelos extratores foram correlacionadas com as características dos solos. Como suporte à execução das análises estatísticas, foram utilizados os softwares Statistica 6.0 e SAEG 5.0.

\section{RESULTADOS E DISCUSSÃO}

\section{Produção de matéria seca da parte aérea}

Não houve influência das doses de Cu na produção de matéria seca da parte aérea (PMSPA) das mudas de eucalipto (Quadro 2). Portanto, a disponibilidade natural desse nutriente nos solos estudados já era adequada ao pleno crescimento das mudas de eucalipto. Os menores teores estimados de $\mathrm{Cu}$ no solo pelo Mehlich-1 foi de 0,12 $\mathrm{mg} \mathrm{dm}^{-3}$ (solo 4) e de 0,09 $\mathrm{mg} \mathrm{dm}^{-3}$ (solo 1) pelo DTPA, para a condição de não adição de $\mathrm{Cu}$ (Quadro 4). Esses teores são bem menores do que aqueles do limite superior da classe de disponibilidade média (nível crítico) usados em Minas Gerais e em São Paulo, ou seja, 1,2 mg dm ${ }^{-3}$ de Cu pelo Mehlich-1 (Alvarez V. et al., 1999) e $0,8 \mathrm{mg} \mathrm{dm}^{-3}$ pelo DTPA (Raij et al., 1997), respectivamente. A baixa demanda de $\mathrm{Cu}$ pelo eucalipto é uma possível explicação para a ausência de resposta das plantas. Mesmo em solução nutritiva, pequenas contaminações do nutriente levam à não obtenção de resposta, conforme relatado por Soares et al. (2000).

Resultados de ausência de resposta de plantas de eucalipto à aplicação de micronutrientes, de modo geral, têm sido frequentemente observados em condições de umidade do solo elevada, próximo à capacidade de campo, em casa de vegetação (Novais et al., 1990). Isso tem ocorrido independentemente de origem do solo, textura, etc. mesmo para aqueles onde, no campo, os sintomas de suas deficiências são evidentes. As limitações por transporte naquelas condições devido ao déficit hídrico e a manutenção de condições para intenso transporte (irrigação frequente) em casa de vegetação são, provavelmente, razões para a diferença de resultados (Novais et al., 1990). 
Quadro 2. Teores de Cu disponível pelo Mehlich-1, Mehlich-3 e DTPA, produção de matéria seca, teores e conteúdos de $\mathrm{Cu}$ na matéria seca de mudas de eucalipto cultivadas em amostras de seis solos de Cerrado (valores médios de quatro repetições), em função das doses de $\mathrm{Cu}$

\begin{tabular}{|c|c|c|c|c|c|c|c|}
\hline \multirow{2}{*}{ Solo } & \multirow{2}{*}{ Dose $\mathbf{C u}$} & \multicolumn{3}{|c|}{ Teor de Cu no solo } & \multirow{2}{*}{$\begin{array}{l}\text { Produção de } \\
\text { MSPA }\end{array}$} & \multirow{2}{*}{$\begin{array}{c}\text { Teor de Cu } \\
\text { na MSPA }\end{array}$} & \multirow{2}{*}{$\begin{array}{c}\text { Conteúdo de } \mathrm{Cu} \\
\text { na MSPA }\end{array}$} \\
\hline & & M-1 & M-3 & DTPA & & & \\
\hline & & 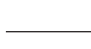 & $\mathrm{ng} \mathrm{dm} \mathrm{m}^{-3}$ & - & g/vaso & $\mathrm{mg} \mathrm{kg}^{-1}$ & $\mu \mathrm{g} / \mathrm{vaso}$ \\
\hline \multirow{5}{*}{1} & 0 & 0,26 & 0,04 & 0,14 & 23,85 & 1,78 & 42,55 \\
\hline & 2 & 1,60 & 1,36 & 1,29 & 19,84 & 6,10 & 120,98 \\
\hline & 4 & 3,17 & 2,90 & 2,79 & 20,60 & 9,02 & 185,74 \\
\hline & 8 & 6,40 & 5,71 & 5,33 & 22,79 & 8,96 & 204,18 \\
\hline & 16 & 11,46 & 12,70 & 10,58 & 21,76 & 9,93 & 215,98 \\
\hline \multirow{5}{*}{2} & 0 & 0,95 & 0,66 & 0,97 & 28,51 & 3,43 & 97,86 \\
\hline & 2 & 1,62 & 1,32 & 1,79 & 29,09 & 4,20 & 122,24 \\
\hline & 4 & 2,56 & 2,61 & 3,17 & 27,14 & 5,50 & 149,21 \\
\hline & 8 & 4,63 & 4,48 & 4,79 & 27,67 & 5,40 & 149,41 \\
\hline & 16 & 9,18 & 8,91 & 10,59 & 28,57 & 6,96 & 198,82 \\
\hline \multirow{5}{*}{3} & 0 & 1,10 & 0,50 & 1,33 & 28,47 & 4,31 & 122,62 \\
\hline & 2 & 2,30 & 1,51 & 1,47 & 27,95 & 5,69 & 159,04 \\
\hline & 4 & 3,77 & 2,64 & 2,83 & 26,60 & 6,24 & 165,86 \\
\hline & 8 & 6,55 & 4,72 & 4,28 & 26,83 & 6,78 & 181,84 \\
\hline & 16 & 12,31 & 8,78 & 8,50 & 27,20 & 7,09 & 192,94 \\
\hline \multirow{5}{*}{4} & 0 & 0,31 & 0,03 & 0,22 & 25,47 & 2,54 & 64,74 \\
\hline & 2 & 1,46 & 1,16 & 1,46 & 27,17 & 6,16 & 167,25 \\
\hline & 4 & 2,86 & 2,13 & 2,88 & 25,34 & 7,12 & 180,52 \\
\hline & 8 & 5,55 & 4,68 & 5,65 & 24,81 & 8,21 & 203,63 \\
\hline & 16 & 11,44 & 9,80 & 11,41 & 24,96 & 9,22 & 230,19 \\
\hline \multirow{5}{*}{5} & 0 & 1,42 & 1,02 & 1,12 & 23,41 & 5,21 & 121,91 \\
\hline & 2 & 2,67 & 1,98 & 2,07 & 23,43 & 5,69 & 133,39 \\
\hline & 4 & 4,13 & 3,15 & 3,56 & 21,42 & 8,28 & 177,42 \\
\hline & 8 & 6,94 & 6,22 & 6,15 & 22,75 & 8,14 & 185,12 \\
\hline & 16 & 12,57 & 11,36 & 11,24 & 21,86 & 8,39 & 183,49 \\
\hline \multirow{5}{*}{6} & 0 & 1,08 & 0,89 & 0,71 & 23,92 & 6,06 & 144,92 \\
\hline & 2 & 2,58 & 2,29 & 1,95 & 22,28 & 7,84 & 174,61 \\
\hline & 4 & 3,85 & 3,87 & 3,17 & 23,34 & 8,12 & 189,49 \\
\hline & 8 & 6,95 & 6,50 & 5,51 & 23,33 & 8,71 & 203,32 \\
\hline & 16 & 13,87 & 12,60 & 10,24 & 22,91 & 11,16 & 255,63 \\
\hline
\end{tabular}

Teor de Cu no solo: Mehlich-1 (M-1), Mehlich-3 (M-3). Matéria seca da parte aérea (MSPA).

Outro aspecto importante é o fato de que, ao crescerem em um ambiente confinado, como de um recipiente, a quantidade de raízes por volume de solo aumenta, reduzindo a distância para a difusão e chegada dos nutrientes à superfície das raízes com o aumento da interceptação radicular. Por isso, mesmo que o teor do nutriente encontrado no solo seja considerado baixo ou, até mesmo, não detectável, pode ser suficiente para a planta crescer e se desenvolver satisfatoriamente, não apresentando ganho de produção de matéria seca com sua aplicação.

De modo geral, uma das condições necessárias para obter ganhos na produção de matéria seca da parte aérea (PMSPA) é um aumento correspondente na quantidade absorvida de um determinado nutriente, que será constituinte da matéria seca produzida adicionalmente. Neste trabalho, como não houve aumento da PMSPA em resposta à aplicação de $\mathrm{Cu}, \mathrm{o}$ aumento dos teores e conteúdos de Cu na matéria seca da parte aérea (MSPA) ocorreu unicamente em resposta ao aumento na aquisição desse nutriente, como teoricamente era esperado. Portanto, em vez de trabalhar com a variável PMSPA, utilizaram-se teores e conteúdos de $\mathrm{Cu}$ na MSPA, os quais podem ser indicativo da resposta positiva da planta à aplicação de $\mathrm{Cu}$.

\section{Teores de $\mathrm{Cu}$ disponível no solo}

Os teores de Cu pelo Mehlich-1, Mehlich-3 e DTPA foram linear e positivamente influenciados pelas doses aplicadas aos solos (Quadro 3). Contudo, a declividade das curvas variou com o solo, indicando a influência de características dos solos utilizados na relação teor recuperado de $\mathrm{Cu}$ em função da dose aplicada (Quadro 3). Isso evidencia diferença quanto à capacidade-tampão de $\mathrm{Cu}$ dos solos e, também, quanto à composição química de cada extrator, com reflexos no modo de extração e na quantidade extraída. As diferenças nos valores dos interceptos das equações que relacionam os teores de acordo com as doses adicionadas (Quadro 3) foram devidas à disponibilidade inicial de $\mathrm{Cu}$ no solo (Quadro 2). Aumentos lineares e positivos nos teores no solo em função de doses, com valores de $\mathrm{R}^{2}$ elevados, foram encontrados por Santos Neto (2003), ao trabalhar com solos de características contrastantes. 
As taxas de recuperação (média dos seis solos) de Cu pelo Mehlich-1, Mehlich-3 e DTPA foram de 69, 64 e $61 \%$ do $\mathrm{Cu}$ adicionado, respectivamente (Quadro 4). Santos Neto (2003) encontrou taxas de recuperação de 67, 61 e 53 \% pelos extratores Mehlich-1, Mehlich-3 e DTPA, respectivamente.

Considerando a ausência de resposta positiva das mudas às doses aplicadas, conclui-se que os teores de $\mathrm{Cu}$ disponível de $0,12 \mathrm{mg} \mathrm{dm}^{-3}$ pelo Mehlich-1 e 0,09 $\mathrm{mg} \mathrm{dm}^{-3}$ de Cu pelo DTPA (Quadro 3) são suficientes para a produção de mudas de eucalipto produzidas em vasos com solo de Cerrado. Para o Mehlich-3, os menores teores estimados (intercepto) para os solos 1 e 4 foram negativos devido aos baixos teores naturais encontrados nesses solos, que foram de $0,04 \mathrm{e}$ $0,03 \mathrm{mg} \mathrm{dm}^{-3} \mathrm{de} \mathrm{Cu}$, respectivamente (Quadro 2).

Os extratores ácidos apresentam maior poder de extração em virtude de sua dissolução ácida, o que causa a extração de frações que não são prontamente disponíveis para as plantas, como, por exemplo, dos óxidos (Cancela et al., 2001; Nascimento, 2001; Ferreira, 2003). No entanto, essas frações não disponíveis no momento da análise do solo poderão sê-lo posteriormente, visto que muitas plantas, a exemplo do eucalipto, apresentam acidificação intensa de sua rizosfera - fenô- meno que pode disponibilizar frações anteriormente inacessíveis às plantas (Novais \& Smyth, 1999).

Em trabalhos como este - que consistem da aplicação de doses de nutrientes ao solo e extração química poucos dias após a aplicação - a interação do nutriente com a matriz sólida do solo é pequena, sendo estreita a relação encontrada entre os teores medidos pelos extratores e as respostas da planta, fato também constatado por Couto (1985) ao trabalhar com doses de Zn. Entretanto, interações mais fortes poderão ocorrer com o passar do tempo, e aquelas boas relações encontradas inicialmente poderão desaparecer. Fato semelhante ocorre com o $\mathrm{P}$, em que ligações adicionais são formadas com o tempo, reduzindo a disponibilidade para a planta (Novais \& Smyth, 1999).

Os teores de $\mathrm{Cu}$ pelos três métodos de extração apresentaram alta correlação entre si (Quadro 4). Resultados concordantes, para os mesmos extratores, foram encontrados por Sequeira (2007) para os solos aqui utilizados, em condições de campo. Cancela et al. (2001) encontraram coeficientes de correlação para os teores de $\mathrm{Cu}$ obtidos por três extratores, para milho e soja, respectivamente de: Mehlich-1 com Mehlich-3 (0,96 e 0,88), Mehlich-1 com DTPA $(0,94$ e 0,84) e DTPA com Mehlich-3 (0,98 e 0,94), para solos de diversas regiões do Estado de São Paulo.

Quadro 3. Equações para teores de Cu disponível (y, $\mathrm{mg} \mathrm{dm}^{-3}$ ) pelo Mehlich-1, Mehlich-3 e DTPA, em função das doses de $\mathrm{Cu}\left(\mathrm{x}, \mathrm{mg} \mathrm{dm}^{-3}\right)$ adicionadas a amostras de seis solos de Cerrado

\begin{tabular}{|c|c|c|c|c|c|c|}
\hline \multirow{2}{*}{ Solo } & \multicolumn{2}{|c|}{ Mehlich-1 } & \multicolumn{2}{|l|}{ Mehlich-3 } & \multicolumn{2}{|l|}{ DTPA } \\
\hline & Equação & $\mathbf{R}^{2}$ & Equação & $\mathrm{R}^{2}$ & Equação & $\mathbf{R}^{2}$ \\
\hline 1 & $\hat{y}=0,33+0,707^{* * *} x$ & 0,997 & $\hat{y}=-0,23+0,794^{* * *} x$ & 0,997 & $\hat{y}=0,09+0,656^{* * *} x$ & 0,999 \\
\hline 2 & $\hat{\mathrm{y}}=0,65+0,523^{* * *} \mathrm{x}$ & 0,996 & $\hat{\mathrm{y}}=0,45+0,523^{* * *} \mathrm{x}$ & 0,997 & $\hat{\mathrm{y}}=0,71+0,608^{* * *} \mathrm{x}$ & 0,997 \\
\hline 3 & $\hat{y}=0,98+0,705^{* * *} \mathrm{x}$ & 0,999 & $\hat{y}=0,53+0,517^{* * *} x$ & 0,999 & $\hat{y}=0,85+0,468^{* * *} \mathrm{x}$ & 0,990 \\
\hline 4 & $\hat{y}=0,12+0,700^{* * * *} x$ & 0,999 & $\hat{y}=-0,13+0,615^{* * *} \mathrm{x}$ & 0,998 & $\hat{y}=0,11+0,703^{* * * t} x$ & 0,999 \\
\hline 5 & $\hat{y}=1,34+0,701^{* * *} \mathrm{x}$ & 0,999 & $\hat{y}=0,78+0,661^{* * *} x$ & 0,998 & $\hat{y}=0,98+0,641^{* * *} x$ & 0,999 \\
\hline 6 & $\hat{\mathrm{y}}=0,86+0,801^{* * *} \mathrm{x}$ & 0,998 & $\hat{\mathrm{y}}=0,85+0,730^{* * *} \mathrm{x}$ & 0,999 & $\hat{\mathrm{y}}=0,75+0,594^{* * *} \mathrm{x}$ & 0,999 \\
\hline
\end{tabular}

Quadro 4. Equações entre teores de Cu disponível pelo Mehlich-1, Mehlich-3 e DTPA em amostras de seis solos de Cerrado

\begin{tabular}{|c|c|c|c|c|c|c|}
\hline \multirow{2}{*}{ Solo } & \multicolumn{2}{|c|}{ Mehlich-3 = f (Mehlich-1) } & \multicolumn{2}{|c|}{ Mehlich-3 = f (DTPA) } & \multicolumn{2}{|c|}{ DTPA $=\mathbf{f}($ Mehlich-1) } \\
\hline & Equação & $\mathbf{R}^{2}$ & Equação & $\mathbf{R}^{2}$ & Equação & $\mathbf{R}^{2}$ \\
\hline 1 & $\hat{y}=-0,57+1,117^{* * *} x$ & 0,989 & $\hat{y}=-0,21+0,925^{* * *} x$ & 0,987 & $\hat{y}=-0,33+1,210^{* * *} x$ & 0,997 \\
\hline 2 & $\hat{y}=0,19+0,998^{* * * *} x$ & 0,998 & $\hat{y}=-0,03+1,159^{* * *} x$ & 0,999 & $\hat{y}=-0,16+0,861^{* * *} \mathrm{x}$ & 0,997 \\
\hline 3 & $\hat{y}=-0,19+0,733^{* * * x} x$ & 0,999 & $\hat{y}=0,19+0,664^{* * * x} x$ & 0,993 & $\hat{y}=-0,37+1,095^{* * *} x$ & 0,990 \\
\hline 4 & $\hat{y}=-0,23+0,877^{* * *} x$ & 0,999 & $\hat{y}=-0,01+1,003^{*+* x} x$ & 0,999 & $\hat{y}=-0,22+0,874^{* * * *} x$ & 0,999 \\
\hline 5 & $\hat{y}=-0,49+0,943^{*+*} x$ & 0,998 & $\hat{y}=-0,25+0,914^{* \star *} x$ & 0,999 & $\hat{y}=-0,23+1,031^{\star * *} x$ & 0,998 \\
\hline 6 & $\hat{\mathrm{y}}=0,07+0,910^{* * * *} \mathrm{x}$ & 0,998 & $\hat{y}=0,13+0,739^{* * *} x$ & 0,997 & $\hat{y}=-0,08+1,229^{* * *} x$ & 0,999 \\
\hline
\end{tabular}

${ }^{\star * * *}$ : significativo a $0,1 \%$ pelo teste F. O y corresponde ao teor de Cu pelo Mehlich-3, nas equações com Mehlich-1 e DTPA; e pelo DTPA, na equação com o Mehlich-1. 
Correlações estreitas e altamente significativas para os teores de $\mathrm{Cu}$ também foram obtidas por Sequeira (2007), que, estudando 150 amostras de solos, encontrou valores de correlação de 0,94 entre Mehlich-1 e Mehlich-3, de 0,82 entre Mehlich-1 e DTPA e de 0,75 entre Mehlich-3 e DTPA. Ferreira (2003), trabalhando com DTPA, Mehlich-1 e Mehlich-3, em 15 amostras de solos de Minas Gerais, observou maior correlação entre DTPA e Mehlich-1 $(r=0,98)$ que entre DTPA e Mehlich-3 $(r=0,74)$ para determinação de $\mathrm{Cu}$.

\section{Relação entre $\mathrm{Cu}$ na parte aérea das mudas e doses aplicadas}

De modo geral, as relações entre os teores e conteúdos de $\mathrm{Cu}$ na matéria seca da parte aérea (MSPA) em função das doses de Cu foram curvilineares, exceto para os conteúdos nos solos 2 e 6 , em que foram lineares (Quadro 5). Os modelos com a forma curvilinear, como o modelo raiz quadrada, o qual apresentou melhor ajuste aos dados obtidos, indicam a forte influência da capacidade-tampão de $\mathrm{Cu}$ do solo, restringindo a aquisição de $\mathrm{Cu}$ pela planta.

Os teores estimados pelas equações na maior dose de $\mathrm{Cu}$ testada $\left(16 \mathrm{mg} \mathrm{dm}^{-3}\right)$ variaram de 6,70 a $11,18 \mathrm{mg} \mathrm{kg}^{-1}$ para os solos 2 e 6 , respectivamente (Quadro 5). O solo 2 possui maior teor de matéria orgânica (MO), e foi justamente nas plantas cultivadas neste solo que se atingiu o menor teor de Cu na MSPA, indicando ser a MO uma característica que tampona esse nutriente no solo, restringindo sua absorção pela planta.

Para diversas culturas, como arroz, cevada, milho, soja e trigo, os valores das correlações lineares entre teores foliares e teores disponíveis no solo têm sido bastante variáveis. Em geral, o extrator adequado para uma cultura não é, necessariamente, o melhor para outras, conforme relatado por vários autores (Cancela et al., 2001; Silva et al., 2003; Gonçalves Jr. et al., 2006; Obrador et al., 2007). Isso indica a necessidade de estudos para cada solo e cultura específica na seleção do extrator mais eficiente.
Portanto, diante dos dados obtidos e devido à similaridade dos valores de $\mathrm{R}^{2}$ dos dois modelos explicativos $($ teor solo $=\mathrm{f}($ dose $)$ e conteúdo planta $=\mathrm{f}($ dose $)$ e do modelo preditivo (teor extrator $\mathrm{i}=\mathrm{f}($ teor extrator $\mathrm{i}+1)$ ), a avaliação da disponibilidade de $\mathrm{Cu}$ para o eucalipto pode ser feita com qualquer um dos três extratores testados. Contudo, como o Mehlich-1 é o extrator utilizado nos laboratórios de análise de rotina em Minas Gerais e em vários Estados do Brasil (Lopes \& Alvarez V., 1999), ele seria mais recomendado, pela sua maior facilidade operacional em relação aos outros e por já ser usado na grande maioria dos laboratórios do País. Entretanto, como mencionado, a extração química poucos dias após a aplicação do nutriente pode não revelar o efeito da forte interação do nutriente com a matriz sólida do solo e, assim, boas relações podem ser encontradas com os três extratores.

Por outro lado, se as extrações forem feitas após um tempo maior de contato do nutriente com o solo, permitindo o estabelecimento de ligações adicionais, poderá haver redução da disponibilidade para a planta e, possivelmente, os extratores apresentarão eficiência diferenciada. Além disso, também é de se esperar que as taxas de recuperação sejam menores do que as encontradas neste trabalho quando se aumenta o tempo de contato do nutriente com o solo. Fato semelhante é o que ocorre com o P (Novais \& Smyth, 1999).

\section{Relação entre teor natural de $\mathrm{Cu}$ no solo e Cu na planta}

De acordo com Bray (1948), o extrator ideal é aquele que extrai as formas disponíveis para as plantas em solos com diferentes características e que apresenta adequação aos laboratórios de rotina. Além disso, devese correlacionar estreitamente com as respostas das culturas à presença dos teores naturais dos nutrientes no solo. Na tentativa de atender a esses preceitos, foram estabelecidas relações comparando o comportamento dos três extratores, nas condições de não aplicação (dose zero) e aplicação dos nutrientes (demais doses testadas), conforme mostram os quadros 6 e 7 .

Quadro 5. Equações de regressão para os teores (y, $\left.\mathrm{mg} \mathrm{kg}^{-1}\right)$ e conteúdos (y, $\left.\mu \mathrm{g} / \mathrm{vaso}\right)$ de Cu na matéria seca

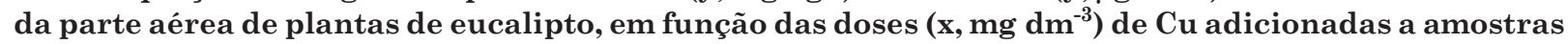
de seis solos de Cerrado

\begin{tabular}{|c|c|c|c|c|c|}
\hline \multirow{2}{*}{ Solo } & \multicolumn{2}{|l|}{ Teor } & \multirow{2}{*}{$\hat{\mathbf{y}}>$} & \multicolumn{2}{|l|}{ Conteúdo } \\
\hline & Equação & $\mathbf{R}^{2}$ & & Equação & $\overline{\mathbf{R}^{2}}$ \\
\hline 1 & $\hat{y}=1,71+4,37^{* *} \sqrt{ } x-0,585^{*} x$ & 0,956 & 9,83 & $\hat{y}=38,4+87,04^{*} \sqrt{ } x-10,475^{\circ} x$ & 0,969 \\
\hline 2 & $\hat{y}=3,48+0,201^{* *} x$ & 0,875 & 6,70 & $\hat{y}=109,3+5,702^{*} x$ & 0,919 \\
\hline 3 & $\hat{y}=4,29+1,23^{+} \sqrt{ } \mathrm{x}-0,130^{\text {ns }} \mathrm{x}$ & 0,998 & 7,13 & $\hat{y}=122,9+27,87^{\square} \sqrt{ } \mathrm{x}-2,587^{\mathrm{ns}} \mathrm{x}$ & 0,996 \\
\hline 4 & $\hat{\mathrm{y}}=2,57+2,90^{*} \sqrt{\mathrm{x}}-0,314^{+} \mathrm{x}$ & 0,999 & 9,15 & $\hat{y}=67,5+76,44^{*} \sqrt{ } x-9,133^{+} x$ & 0,989 \\
\hline 5 & $\hat{y}=5,18+0,656^{*} x-0,029^{\circ} x^{2}$ & 0,845 & 8,25 & $\hat{y}=119,5+13,66^{\circ} x-0,606^{+} x^{2}$ & 0,912 \\
\hline 6 & $\hat{y}=6,72+0,279^{* *} x$ & 0,927 & 11,18 & $\hat{y}=155,5+6,350^{* *} x$ & 0,964 \\
\hline
\end{tabular}

ns $,{ }^{\circ},+\stackrel{\circ}{*}, \mathrm{e}^{* *}$ : não significativo e significativo a $20,15,10,5$, e $1 \%$ pelo teste $\mathrm{F}$, respectivamente. $\hat{\mathrm{y}}>=$ teor estimado para a dose maior $\left(16 \mathrm{mg} \mathrm{dm}^{-3}\right)$. 
Quadro 6. Equações de regressão entre PMSPA, teores e conteúdos de Cu na MSPA de mudas de eucalipto cultivadas em amostras de seis solos de Cerrado, em função dos teores naturais existentes no solo (dose zero) extraídos por Mehlich-1, Mehlich-3 e DTPA

\begin{tabular}{|c|c|c|c|c|c|c|}
\hline & \multicolumn{2}{|l|}{ PMSPA (g/vaso) } & \multicolumn{2}{|l|}{ Teor na planta (mg kg $\left.{ }^{-1}\right)$} & \multicolumn{2}{|l|}{ Conteúdo na planta ( $\mu$ g/vaso) } \\
\hline & Equação & $\mathbf{R}^{2}$ & Equação & $\mathbf{R}^{2}$ & Equação & $\mathbf{R}^{2}$ \\
\hline M-1 & $\hat{y}=-0,17+68,59^{\circ} \sqrt{ } x-41,02^{\circ} x$ & 0,51 & $\hat{y}=1,33+2,965^{*} \mathrm{x}$ & 0,74 & $\hat{y}=35,92+73,087^{* *} x$ & 0,81 \\
\hline M-3 & $\hat{y}=19,18+38,18^{*} \sqrt{x}-34,17^{*} x$ & 0,85 & $\hat{y}=2,02+3,506^{* *} x$ & 0,84 & $\hat{y}=55,24+81,969^{* * *} x$ & 0,82 \\
\hline DT & $\hat{y}=23,95+2,336^{\mathrm{ns}} \mathrm{x}$ & 0,19 & $\hat{y}=-6,22+27,11^{\circ} \sqrt{x}-3,025^{+} x$ & 0,73 & $\hat{y}=-132,7+593,7^{\circ} \sqrt{x}-338,63^{\circ} \mathrm{x}$ & 0,86 \\
\hline
\end{tabular}

ns $+{ }^{\circ},{ }^{*}$ e ${ }^{* *}$ : não significativo e significativo a $15,10,5$ e $1 \%$ pelo teste F, respectivamente. M-1: Mehlich-1; M-3: Mehlich-3; DT: DTPA; PMSPA: produção de matéria seca da parte aérea; MSPA: matéria seca da parte aérea.

Quadro 7. Coeficiente de correlação linear (r) entre teores no solo (Mehlich-1, Mehlich-3 e DTPA) e conteúdos de Cu na matéria seca da parte aérea de mudas de eucalipto cultivadas em amostras de seis solos de Cerrado na dose zero e nas demais doses

\begin{tabular}{|c|c|c|c|c|c|c|}
\hline \multirow{2}{*}{ Dose } & \multicolumn{3}{|c|}{ Teor disponível no solo com teor na planta } & \multicolumn{3}{|c|}{ Teor disponível no solo com conteúdo na planta } \\
\hline & Mehlich-1 & Mehlich-3 & DTPA & Mehlich-1 & Mehlich-3 & DTPA \\
\hline Zero & $0,84^{* * *}$ & $0,84^{* * *}$ & $0,60^{* * *}$ & $0,87^{* * *}$ & $0,82^{* * *}$ & $0,68^{* * *}$ \\
\hline Demais $^{(1)}$ & $0,66^{* * *}$ & $0,69^{* * *}$ & $0,61^{* * * *}$ & $0,77^{* * *}$ & $0,77^{* * *}$ & $0,74^{* * *}$ \\
\hline
\end{tabular}

(1) Demais: doses 2, 4, 8 e $16 \mathrm{mg} \mathrm{dm}{ }^{-3}$ de $\mathrm{Cu} .{ }^{* * *}$ : significativo a $0,1 \%$ pelo teste $\mathrm{F}$.

Os teores de $\mathrm{Cu}$ pelo Mehlich-3 apresentaram melhor ajuste no modelo que relaciona a PMSPA e o teor na planta em função dos teores naturais disponíveis (dose zero) (Quadro 6).

É sabido que o nível crítico de um determinado nutriente no solo é variável com a capacidade-tampão desse nutriente no solo (Novais \& Smyth, 1999), e ter um nível crítico independente das características que conferem a capacidade-tampão do solo a um nutriente certamente não é o procedimento mais adequado. Mesmo assim, para esse restrito grupo de seis solos, com as equações do quadro 6 é possível calcular um único nível crítico independente do solo: por meio da primeira derivada encontra-se o ponto de máximo (PMSPA máxima - Y máx); substituindo-o na equação, acha-se o valor de $\mathrm{X}$ (teor de $\mathrm{Cu}$ disponível no solo) correspondente ao Y máx, e o valor é de 0,71 e $0,31 \mathrm{mg} \mathrm{dm}^{-3} \mathrm{de} \mathrm{Cu}$, respectivamente pelos extratores Mehlich-1 e Mehlich-3. Esses resultados, contrastantes com aqueles sugeridos do quadro 3 , mostram a importância e a influência que o método pode ter nos resultados do estudo.

Os coeficientes de correlação entre os teores de $\mathrm{Cu}$ disponíveis no solo com os teores e conteúdos nas plantas foram mais elevados para todos os extratores na dose zero que nas demais doses (Quadro 7). Os extratores Mehlich-1 e Mehlich-3 mostraram valores das correlações próximos entre si, porém mais elevados que os do DTPA.

\section{Características do solo e recuperação de $\mathrm{Cu}$ pelos extratores}

Os coeficientes de correlação entre as taxas de recuperação de $\mathrm{Cu}$ pelos extratores e os teores de matéria orgânica foram altamente significativos para o Mehlich-1 (M-1) e Mehlich-3 (M-3) e não significativos para o DTPA (Quadro 8), indicando que os extratores M-1 e M-3 extraem mais $\mathrm{Cu}$ de solos com menores teores de matéria orgânica, pois menos sítios de adsorção são encontrados nesses solos. As correlações com teor de argila e $\mathrm{P}$ remanescente $(\mathrm{P}$ rem) foram mais elevadas com o M-3. Isso se deve provavelmente ao menor desgaste dos extratores ácidos nos solos arenosos, que, de modo geral, possuem menores teores de MO. O desgaste é um efeito relatado com frequência na extração de $\mathrm{P}$ do solo por extratores ácidos (Novais \& Smyth, 1999).

A matéria orgânica do solo tem alta afinidade pelo $\mathrm{Cu}$ e forma complexos orgânico-metálicos bastante estáveis, que dificultam a extração desse nutriente (Stevenson, 1991). O extrator DTPA atua por complexação; com isso, sua capacidade de extração independe do teor de matéria orgânica e argila do solo - complexantes mais fracos que o DTPA. Embora com valores mais baixos, as correlações com $\mathrm{H}+\mathrm{Al}$ seguiram a mesma tendência daquelas com o teor de matéria orgânica, pois são características do solo correlacionadas entre si. 
As correlações com P-rem foram significativas e positivas para M-1 e M-3, com valores mais altos do que aqueles encontrados com o teor de argila. O Prem é uma característica que tem sido usada como medida do fator capacidade de $\mathrm{P}$ e de $\mathrm{S}$ (Alvarez V. et al., 2000). Os valores de correlações observados neste trabalho indicam que o P-rem reflete a capacidade de adsorção de $\mathrm{Cu}$ pelo solo. Comparado com o teor de argila, o P-rem apresenta melhor correlação com as taxas de recuperação pelo M-1 e M-3, especialmente com este último. Além disso, é uma análise mais simples e de menor custo do que a de argila. O P-rem e o teor de argila são características do solo também correlacionadas entre si; de modo geral, para os solos de regiões tropicais altamente intemperizados, o Prem diminui com o aumento do teor de argila.

As correlações com o pH foram altamente significativas e negativas para os três extratores, indicando que a extração diminui com o aumento do $\mathrm{pH}$ do solo (Quadro 8).

As correlações entre o melhor indicador de disponibilidade, a planta, e seus teores e conteúdos relacionados com as características do solo apresentaram sinais concordantes com aquelas do Mehlich-1 e Mehlich-3 (Quadro 8), indicando que a planta é sensível à capacidade-tampão de $\mathrm{Cu}$, e esses extratores também o foram. O DTPA apresentou melhor correlação com o pH, indicando ser o extrator mais sensível a diferentes condições de $\mathrm{pH}$, diminuindo a extração de $\mathrm{Cu}$ à medida que se elevaram os valores de $\mathrm{pH}$, o que corrobora os dados obtidos por Nascimento (2001) e Santos Neto (2003).
Equações de regressão múltipla foram ajustadas entre as taxas de $\mathrm{Cu}$ recuperado em função das doses adicionadas aos solos e algumas características dos solos que influenciam na capacidade-tampão de $\mathrm{Cu}$ (Quadro 9).

Os modelos de regressão ajustados para as taxas de $\mathrm{Cu}$ recuperado em função do adicionado (Quadro 9) mostram que a matéria orgânica (MO) prediz 89,5 \% dos teores de $\mathrm{Cu}$ recuperados pelo Mehlich-1. Para Mehlich-3, o P remanescente (P-rem) justifica 96,1 \% da variação do $\mathrm{Cu}$ recuperado. As taxas de recuperação do Cu extraído pelo DTPA são explicadas em $97,7 \%$ pelo $\mathrm{pH}$.

Com base nos modelos ajustados, as variáveis de solo que melhor explicam as taxas de recuperação do Cu pelos extratores Mehlich-1 e Mehlich-3 são matéria orgânica, P-rem e teor de argila. Pelo DTPA, o pH é a melhor variável explicativa das taxas de recuperação por este extrator. As taxas de recuperação de Cu para os mesmos três extratores deste trabalho foram mais bem explicadas pelos teores de argila e de matéria orgânica (Santos Neto, 2003).

\section{CONCLUSÕES}

1. Os níveis críticos de $\mathrm{Cu}$ em solos de Cerrado, para o crescimento de mudas de eucalipto, são iguais ou inferiores a 0,12 e $0,09 \mathrm{mg} \mathrm{dm}^{-3}$, pelos extratores Mehlich-1 e DTPA, respectivamente.

Quadro 8. Coeficientes de correlação linear (r) entre características do solo, teor e conteúdo de Cu na MSPA ${ }^{(1)}$ e as declividades das equações do quadro 3 - $\mathrm{Cu}$ recuperado pelos extratores Mehlich-1(M-1), Mehlich-3 (M-3) e DTPA, em função do adicionado às amostras de seis solos de Cerrado

\begin{tabular}{|c|c|c|c|c|c|}
\hline \multirow{2}{*}{ Característica do solo } & \multicolumn{3}{|c|}{ Extratores } & \multicolumn{2}{|c|}{ Planta } \\
\hline & M-1 & M-3 & DTPA & Teor & Conteúdo \\
\hline $\mathrm{pH}$ & $-0,61^{* * *}$ & $-0,70^{* * *}$ & $-0,71^{* * *}$ & $-0,31 \mathrm{~ns}$ & $-0,12^{\mathrm{ns}}$ \\
\hline $\mathrm{H}+\mathrm{Al}$ & $-0,44^{* *}$ & $-0,57^{* * *}$ & $0,30^{\mathrm{ns}}$ & $-0,29 \mathrm{~ns}$ & $-0,12^{\mathrm{ns}}$ \\
\hline Matéria orgânica & $-0,85^{* * *}$ & $-0,85^{* * *}$ & $0,04^{\mathrm{ns}}$ & $-0,42 \mathrm{~ns}$ & $-0,19 \mathrm{~ns}$ \\
\hline P-rem & $0,59^{* * *}$ & $0,98^{* * *}$ & $0,45^{* *}$ & $0,38 \mathrm{~ns}$ & $0,13^{\mathrm{ns}}$ \\
\hline Argila & $-0,42 \mathrm{~ns}$ & $-0,88^{* * *}$ & $-0,50^{* *}$ & $-0,27 \mathrm{~ns}$ & $-0,07 \mathrm{~ns}$ \\
\hline
\end{tabular}

${ }^{(1)}$ MSPA: matéria seca da parte aérea. ${ }^{\mathrm{ns}},{ }^{* *} \mathrm{e}^{* * *}$ : não significativo e significativo a 1 e $0,1 \%$ pelo teste $\mathrm{F}$, respectivamente.

Quadro 9. Equações de regressão para as taxas de recuperação $\left(\mathrm{mg} \mathrm{dm}^{-3} / \mathrm{mg} \mathrm{dm}^{-3}\right) \mathrm{de}$ Cu por Mehlich-1 (M-1), Mehlich-3 (M-3) e DTPA, em função de algumas características de seis solos de Cerrado

\begin{tabular}{lcllll}
\hline Equação Mehlich-1 & $\mathbf{R}^{2}$ & Equação Mehlich-3 & $\mathbf{R}^{2}$ & \multicolumn{1}{c}{ Equação DTPA } & $\mathbf{R}^{2}$ \\
\hline TR $=0,99-0,0906^{* * *} \mathrm{MO}$ & 0,895 & $\mathrm{TR}=0,36+0,0114^{* * *} \mathrm{P}-\mathrm{rem}$ & 0,961 & $\mathrm{TR}=8,09-2,797^{* *} \mathrm{pH}+0,260^{* *} \mathrm{pH}^{2}$ & 0,977 \\
$\mathrm{TR}=0,50+0,0083^{*} \mathrm{P}-\mathrm{rem}$ & 0,594 & $\mathrm{TR}=0,92-0,0063^{* *} \mathrm{ARG}$ & 0,775 & $\mathrm{TR}=0,96+0,01795^{*}(\mathrm{H}+\mathrm{Al})-0,0849^{*} \mathrm{pH}$ & 0,778 \\
$\mathrm{TR}=0,91-0,0046^{\circ} \mathrm{ARG}$ & 0,470 & $\mathrm{TR}=0,92-0,0869^{*} \mathrm{MO}$ & 0,715 & $\mathrm{TR}=1,09-0,0907^{*} \mathrm{pH}$ & 0,618 \\
\hline
\end{tabular}

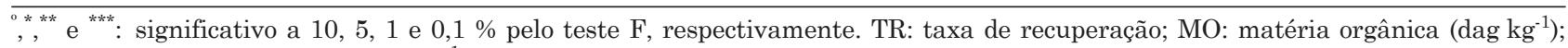
P-rem: fósforo remanescente $\left(\mathrm{mg} \mathrm{L}^{-1}\right)$ e ARG: argila (\%). 
2. Os teores de $\mathrm{Cu}$ no solo e na planta, bem como o respectivo conteúdo, aumentam com a elevação das doses adicionadas desse nutriente.

3. Os teores e conteúdos de Cu na planta mostram estreita relação com os teores de $\mathrm{Cu}$ no solo pelos três extratores.

4. Os teores de $\mathrm{Cu}$ pelo Mehlich-1, Mehlich-3 e DTPA são altamente correlacionados entre si.

5. A avaliação da disponibilidade de $\mathrm{Cu}$ para mudas de eucalipto pode ser feita com qualquer um dos três extratores. Entretanto, o Mehlich-1 é o mais recomendado, pela facilidade operacional deste método em relação aos outros e por ele já ser usado na grande maioria dos laboratórios do País.

6. O teor de matéria orgânica e o P-remanescente são características dos solos que mais influenciam negativamente as taxas de recuperação de $\mathrm{Cu}$ pelos extratores Mehlich-1 e Mehlich-3, enquanto para o DTPA as taxas de recuperação são mais influenciadas pelo $\mathrm{pH}$.

\section{LITERATURA CITADA}

ALVAREZ V., V.H. Equilíbrio de formas disponíveis de fósforo e enxofre em dois Latossolos de Minas Gerais. Viçosa, MG, Universidade Federal de Viçosa, 1974. 125p. (Tese de Mestrado)

ALVAREZ V., V.H.; NOVAIS, R.F.; BARROS, N.F.; CANTARUTTI, R.B. \& LOPES, A.S. Interpretação dos resultados das análises de solos. In: RIBEIRO, A.C.; GUMARÃES, P.T.G. \& ALVAREZ V., V.H., eds. Recomendações para o uso de corretivos e fertilizantes em Minas Gerais. Viçosa, MG, CFSEMG, 1999. p.25-32.

ALVAREZ V., V.H.; NOVAIS, R.F.; DIAS, L.E. \& OLIVEIRA J.A. Determinação e uso do fósforo remanescente. B. Inf. SBCS, 25:27-32, 2000.

BRAY, R.H. Requirements for successful soils tests. Soil Sci., 66:83-89, 1948 .

BOUCHARDET, J.A. Crescimento características físicas e anatômicas da madeira juvenil de dois clones de Eucalyptus grandis Hill ex Maiden em resposta à aplicação de boro. Piracicaba, ESALQ, 2002. 69p. (Tese de Mestrado)

CANCELA, R.C.; FREIRE, A.R.; ABREU, C.A. \& GONZÁLEZ, A.P. Eficacia de cuatro extractantes an la evaluación de la disponibilidad de cobre para maíz y soja. Bragantia, 60:205-212, 2001.

COUTO, C.; NOVAIS, R.F.; BARROS, N.F. \& NEVES, J.C.L. Resposta do eucalipto à aplicação de zinco em amostras de solos de cerrado. R. Árvore, 9:134-148, 1985.

DELL, B.; MALAJCZUK, D.; XU, D. \& GROVE, T.S. Nutrient disorders in plantation eucalypts. 2.ed. Canberra, ACIAR, 2001. 188p
FERREIRA, G.B. Dinâmica das frações de micronutrientes catiônicos e esgotamento de formas disponíveis de boro, cobre, ferro, manganês e zinco, em solos de Minas Gerais. Viçosa, MG, Universidade Federal de Viçosa, 2003, 169p. (Tese de Doutorado)

GONÇALVES Jr., A.C.; PRESTES, A.L.; RIBEIRO, O.L. \& SANTOS, A.L. Métodos extratores e fitodisponibilidade de zinco para milho em Argissolo Vermelho-Amarelo eutrófico. Sci. Agr., 7:35-40, 2006.

LINDSAY, W.L. \& NORVELL, W.A. Developemnt of a DTPA soil of zinc, iron, manganese and copper. Soil Sci. Soc. Am. J., 42:421-428, 1978

LOPES, A.S. \& ALVAREZ V., V.H. Apresentação dos resultados das analises de solo. In: RIBEIRO, A.C.; GUIMARÃES, P.T.G. \& ALVAREZ V., V.H., eds. Recomendação para o uso de corretivos e fertilizantes em Minas Gerais. Viçosa, MG, CFSEMG, 1999. p.21-24.

MARSCHNER, H. Mineral nutrition of higher plants. 2.ed. San Diego, Academic Press, 1995. 889p.

MEHLICH, A. Determination of $\mathrm{P}, \mathrm{Ca}, \mathrm{Mg}, \mathrm{K}, \mathrm{Na}$ and $\mathrm{NH}_{4}$. Raleigh, North Carolina Soil Test Div., 1953. (não publicado)

MEHLICH, A. Mehlich-3 soil test extractant: A modification of Mehlich-2 extractant. Comm. Soil Sci. Plant Anal., 15:1409-1416, 1984.

NASCIMENTO, C.W.A. Dessorção, extração e fracionamento de zinco, cobre e manganês em solos. Viçosa, MG, Universidade Federal de Viçosa, 2001. 60p. (Tese de Doutorado)

NOVAIS, R.F.; BARROS, N.F. \& NEVES, J.C.L. Nutrição mineral do eucalipto. In: BARROS, N.F. \& NOVAIS, R.F., eds. Relação solo-eucalipto. Viçosa, MG, Universidade Federal de Viçosa, 1990. p.25-98.

NOVAIS, R.F. \& SMYTH, T.J. Fósforo em solo e planta em condições tropicais. Viçosa, MG, Universidade Federal de Viçosa, 1999. 399p.

OBRADOR, A.; ALVAREZ, J.M.; LOPEZ-VALDIVIA, L.M.; GONZALEZ, D.; NIVILLO, M.I. \& RICO, M.I. Relationships of soil properties with $\mathrm{Mn}$ and $\mathrm{Zn}$ distribution in acid soils and their uptake by a barley crop. Geoderma, 137:432-443, 2007.

PINHEIRO, A.L. Reflexos da fertilização mineral de boro na estrutura anatômica, no crescimento e na seca-deponteiro de Eucalyptus citriodora Hook e Eucalyptus camaldulensis Dehn no Cerrado de Minas Gerais. Curitiba, Univrsidade Federal do Paraná, 1999. 191p. (Tese de Doutorado)

RAIJ, B.van.; CANTARELLA, H.; QUAGGIO, J.A. \& FURLANI, Â.M.C. Recomendações de adubação e calagem para o Estado de São Paulo. Campinas: IAC, 1996. 285p.

RUIZ, H.A. Incremento da exatidão da análise granulométrica do solo por meio da coleta da suspensão (silte + argila). $R$. Bras. Ci. Solo, 29:297-300, 2005.

SANTOS NETO, J.A. Taxas de recuperação de zinco, cobre e boro por diferentes extratores em solos da Bahia e Minas Gerais. Viçosa, MG, Universidade Federal de Viçosa, 2003. 51p. (Tese de Mestrado) 
SEQUEIRA, C.H. Disponibilidade de micronutrientes em solos e sua correlação com teores foliares em povoamentos jovens de eucalipto. Viçosa, MG, Universidade Federal de Viçosa, 2007. 36p. (Tese de Mestrado)

SILVA, M.A.G.; MUNIZ, A.S.; MATA, J.D.V. \& CEGANA, A.C.V Extratores para a quantificação do zinco e do cobre em solos cultivados com soja. Acta Sci. Agron., 26:361-366, 2003.
SOARES, C.R.F.S.; SIQUEIRA, J.O.; CARVALHO, J.G.; MOREIRA, F.M.S. \& GRAZZIOTTI, P.H. Crescimento e nutrição mineral de Eucalyptus maculata e E. urophylla em solução nutritiva com concentração crescente de cobre. R. Bras. Fisiol. Veg., 12:213-255, 2000.

STEVENSON, F.J. Organic matter-micronutrient reactions in soil. In: MORTVEDT, J.J.; COX, F.R.; SHUMAN, L.M. \& WELCH, R.M., eds. Micronutrients in agriculture. 2.ed. Madison, Soil Science Society of Agronomy, 1991. p.145181. 